Théologiques

Théologiques

\title{
La prise en compte des dimensions religieuse et spirituelle dans l'intervention psychosociale
}

\section{François Lefebvre}

Volume 9, numéro 2, automne 2001

Sens et spiritualité dans les pratiques professionnelles

URI : https://id.erudit.org/iderudit/007296ar

DOI : https://doi.org/10.7202/007296ar

Aller au sommaire du numéro

Éditeur(s)

Faculté de théologie de l'Université de Montréal

ISSN

1188-7109 (imprimé)

1492-1413 (numérique)

Découvrir la revue

Citer cet article

Lefebvre, F. (2001). La prise en compte des dimensions religieuse et spirituelle dans l'intervention psychosociale. Théologiques, 9(2), 69-93.

https://doi.org/10.7202/007296ar d'utilisation que vous pouvez consulter en ligne.

https://apropos.erudit.org/fr/usagers/politique-dutilisation/ 


\section{La prise en compte des dimensions religieuse et spirituelle dans l'intervention psychosociale}

François LeFebVRE, M.A., M.Ps. Psychologue

L'intervenant psychosocial, qu'il soit psychologue, travailleur social, médecin, ou infirmière, a de bonnes chances d'avoir à interagir avec le religieux dans le cadre de son travail. Par ailleurs, il est reconnu que la question religieuse est délicate, qu'elle peut susciter des réactions autant chez l'intervenant que chez le patient et ainsi compromettre la démarche de relation d'aide ${ }^{1}$. Dans cet article, je tenterai de démontrer l'importance, pour l'intervenant psychosocial, de se familiariser avec la dimension religieuse, quelle que soit sa profession ou le cadre dans lequel il exerce. Le contexte de sécularisation et de pluralisme religieux m'amène à situer l'émergence de la question religieuse dans l'intervention au niveau de la culture, en plus d'en souligner la possible pertinence, ce qui contraste avec la traditionnelle animosité des milieux « psy » face à la religion. La foi religieuse doit donc être située dans le cadre plus large du respect de la diversité culturelle et de l'aspect thérapeutique des dimensions spirituelle et religieuse. Pour illustrer ce nouveau contexte de pluralisme, évoquons seulement qu'il est de plus en plus probable que se trouvent des intervenants de la santé ressentant une même incompréhension et un même sentiment d'étrangeté devant les dévotions à la Vierge Marie d'une dame catholique âgée, que devant les ablutions rituelles d'un musulman. Je propose donc que les intervenants se familiarisent davantage avec la question religieuse pour qu'ils puissent la prendre en compte au besoin, de la même façon qu'ils pren-

1. M. ZEIGER et J.E. LEWIS, "The Spirituality Responsible Therapist. Religious Material in the Psychotherapeutic Setting ", Psychotherapy, 35 (1998) p. 415-424. 
nent en compte l'origine culturelle de leur patient avant de poser un diagnostic et surtout avant d'élaborer un plan de traitement.

Pour aborder la façon dont se posent les questions religieuses ou spirituelles au sein des pratiques psychosociales des intervenants, cet article s'attachera à l'examen des différentes positions que peut adopter l'intervenant et la nécessité pour lui d'être au clair avec l'ancrage culturel de ses propres pratiques. En terminant, je dégagerai des situations types dans lesquelles peuvent émerger les questions religieuse et spirituelle.

\section{La question religieuse à travers le prisme de l'approche multiculturelle}

Le milieu de la santé a déjà eu à réagir et à se positionner face au multiculturalisme de la clientèle à desservir. On observe à cet égard que les institutions adhèrent aux principes d'uniformité, d'universalité (mêmes services pour tous) et de neutralité (séparation Église-État) afin de faciliter la gestion et d'assurer l'équité 2 . Mais selon Bibeau, cette attitude s'est révélée une façon de renforcer l'identité civile commune au détriment des identités ethnique, religieuse, ou linguistique des groupes particuliers ${ }^{3}$. Citant Charles Taylor, il se demande comment il est possible pour une société de traiter tous ses membres comme égaux si elle ne prend pas en considération l'identité culturelle singulière des citoyens. Des études démontrent d'ailleurs l'effet négatif d'un oubli de sa propre culture pour adopter celle de la majorité. À titre d'exemple, Green ${ }^{4}$ rapporte celles portant sur des étudiants originaires du Sud-Est asiatique et qui étudient dans des écoles américaines. Ces études révèlent que ceux qui sont le plus acculturés aux normes de la vie américaine ont des notes inférieures à ceux qui sont moins acculturés. Selon Green, ces données suggèrent que bien des gens y gagneraient à garder leurs distances face à la culture principale ${ }^{5}$ ou, pourrions-nous ajouter,

2. G. BIBEAU, «Le pluralisme culturel dans les services publics, recadrage anthropologique de la société postmoderne ", conférence d'ouverture du colloque Le pluralisme, défi d'aujourd'bui dans le réseau de la santé et des services sociaux, Montréal, Ministère de la Santé et des Services Sociaux, 1992. 3. BIBEAU, « Le pluralisme culturel », p. 20.

4. J.W. GReEN, Cultural Awareness in the Human Services. A MultiEthnic Approach, Boston, Allyn and Bacon, 199933, p. 22.

5. GREEN, Cultural Awareness, p. 22. 
à rester attachés ou à renouer avec leurs propres racines culturelles ou religieuses, tout en s'intégrant dans la société d'accueil.

Plutôt qu'une approche d'assimilation, Bibeau prône une approche respectueuse des individualités et de leur culture d'appartenance. Cette approche se distingue de l'approche américaine qui se limite à renvoyer la différenciation culturelle à sa périphérie et elle se distingue aussi de l'approche française où l'immigrant sait qu'il devra progressivement se délester de sa culture d'origine et se soumettre à la laïcité des institutions publiques pour devenir citoyen d'une "république une et indivisible ${ }^{6}$. Cette approche pluraliste se fonde sur l'irrecevabilité du dogme médical selon lequel il existerait un corps biologique de nature transculturelle et universelle qui serait totalement indépendant de la culture des personnes. Elle reconnaît plutôt " qu'à travers la médiation du corps, le monde des valeurs d'un groupe rejoint la vie la plus intime des personnes individuelles, rendant celles-ci en quelque sorte prisonnières d'une certaine conception de la vie, de la souffrance et de la mort qui refait surface lorsqu'une personne tombe malade ou souffre d'un problème ${ }^{7}$ ». Sur ce plan, l'anthropologie médicale a beaucoup fait pour éviter d'isoler la maladie de tant d'aspects de la vie sociale. Kleinman est un de ceux-là ${ }^{8}$, de même que Henri Collomb qui s'est distingué par son étude des ressources de guérison locales à Dakkar. Ce dernier est reconnu pour son intuition de la nécessité irréductible, hic et nunc, d'une approche résolument "culturelle» du désordre mental ${ }^{9}$. Par extension, je ne crois pas que nous puissions exclure de cette approche culturelle la prise en compte des croyances religieuses de la personne souffrante.

\section{Les croyances religieuses : un fait de la culture}

Les croyances religieuses sont une dimension de la culture. Elles contribuent à façonner la vision qu'ont les gens du monde dans lequel ils vivent. Elles sont d'autant plus prégnantes qu'elles sont partagées par

6. BIBEAU, « Le pluralisme culturel », p. 29.

7. BIBEAU, « Le pluralisme culturel », p. 39.

8. Le lecteur intéressé à en savoir plus long sur les nombreux ouvrages de Kleinman est invité à consulter l'ouvrage de J. W. Green cité précédemment, qui propose un modèle à partir des travaux de celui-ci (p. 52).

9. M. SIMON, Les maux de l'autre. La maladie comme objet anthropologique, Paris, L'Harmattan, 1994, p. 30. 
un groupe et confirmées par l'expérience. Elles sont alors reconnues comme vraies, bien que parfois illogiques. La culture et la religion sont porteuses de diverses théories de la causalité qu'il peut être utile d'explorer dans une intervention. Ainsi à quelles causes un patient attribue-t-il le trouble ou la maladie dont il souffre ? À des causes d'ordre naturelle, supranaturelle, animiste ou magique ? Il est intéressant pour l'intervenant de garder cela en tête lors de l'évaluation car les patients vont agir selon ce qu'ils croient être la cause de leur mal, alors que ça ne correspond pas nécessairement au diagnostic de l'intervenant ${ }^{10}$. Bien avant d'être allé chercher un avis professionnel, le futur patient a articulé une vision de ce qui est normal et de ce qui ne va pas. Il a comparé sa détresse avec la connaissance et la pratique d'autres personnes et en est venu à une formulation culturelle de ce qu'est le problème et de ce qu'il peut faire avec. Selon Green ${ }^{11}$, tout cela constitue la recherche personnelle pour donner un «sens » à l'expérience de souffrance. On peut voir la culture comme tout ce qui est intervenu dans cette recherche : les idées, les croyances, les réponses, les conversations, la sagesse partagée, les gestes corporels, les émotions soulevées, les solutions tentées, les espoirs et les attentes revues, et même les invocations du surnaturel.

\section{Dans les institutions de santé, ça ne va plus de soi}

Avec la laïcisation de nos institutions de santé, le fait religieux relève moins de l'État et des grandes Églises que de la sphère privée et civile. Il est vrai que la législation exige de chaque établissement de santé qu'il adopte un règlement sur l'organisation du service de pastorale ${ }^{12}$. L'article 100 de la loi 120 adoptée en 1991 stipule que :

Les établissements ont pour fonction d'assurer la prestation de services de santé ou de services sociaux de qualité, qui soient continus, accessibles et respectueux des droits des personnes et de leurs besoins spirituels

10. H. ToumisheY, "Multicultural Health Care ", dans R. MASI, L. MENSAH et K.A. MCLEOD, dir., Health and Cultures. Exploring the Relationships, Oakville, Ont., Mosaic Press, 1993, p. 120.

11. GREEN, Cultural Awareness, p. 51.

12. Ministère de la Santé et des Services Sociaux, Pastorale en établissements de santé et de services sociaux. Rapport du Comité de travail, Québec, 1997, révisé en mai 2000, p. 17. 
et qui visent à réduire ou à solutionner les problèmes de santé et de bienêtre et à satisfaire les besoins des groupes de la population...

La loi se veut inclusive en élargissant les besoins religieux aux "besoins spirituels » et en n'exigeant pas d'entente avec les autorités religieuses. La dimension religieuse nommée en terme de spiritualité devient davantage une dimension de la vie du patient dont on doit tenir compte dans la mesure où son occultation pourrait compromettre le succès du traitement. On passe alors d'un religieux qui allait de soi et qui était peut-être devenu pour les gestionnaires un droit acquis à respecter, à un aspect de la culture du patient à intégrer dans les soins, une dimension de l'être humain à prendre au sérieux.

En ce sens un médecin spécialiste en région déplorait récemment la transformation, au milieu des années 90, de la chapelle en fumoir. Le lieu de recueillement était alors confiné à une surface réduite du tiers et séparée du fumoir par un épais rideau qui ne suffisait pas à préserver ce lieu de l'odeur du tabac. "Des familles venant de vivre le décès d'un proche s'y sont retrouvées toutes tassées et choquées de devoir vivre des moments si douloureux dans un tel environnement, dit-il. On investit des millions dans la technologie mais absolument rien dans l'aménagement d'un lieu où les patients et leurs proches pourraient aller se recueillir, continue-t-il. C'est d'autant plus inacceptable que la maladie est souvent un moment où les gens renouent avec leur tradition spirituelle et y recherchent spontanément un appui. »

Cet exemple témoigne que la dimension religieuse n'est pas nécessairement prise en compte automatiquement de par le statut et la tradition des institutions de santé mais qu'elle doit être considérée à partir d'une politique de santé et d'une vision globale du traitement. De la même façon qu'on tente d'avoir une approche ouverte aux diverses cultures, la question religieuse doit aussi être considérée dans le plan de traitement. Elle ne va plus de soi au plan institutionnel, mais elle demeure souvent une dimension de la vie des patients, de leurs familles et de leur entourage. Attardons-nous maintenant au positionnement de l'intervenant face au phénomène religieux. 


\section{La prise en compte du religieux par l'intervenant : diverses conceptions}

Les institutions peuvent avoir des plans qui considèrent et intègrent la diversité culturelle et religieuse, mais en bout de ligne c'est l'intervenant qui permet ou non l'exploration des dimensions spirituelle et religieuse et le recours à celles-ci lorsqu'il le juge aidant. Nous verrons dans un premier temps le préjugé négatif légendaire dont a été l'objet la croyance religieuse dans le domaine de la psychothérapie pour ensuite présenter trois positions typiques possibles de l'intervenant face à la croyance religieuse. Ces réflexions nous paraissent éclairer plusieurs types de relations professionnelles, tant dans les milieux hospitaliers, psychosociaux que communautaires.

\subsection{Un préjugé négatif légendaire renforcé par une psychanalyse égarée}

La traditionnelle neutralité thérapeutique face à la croyance religieuse contraste avec les réactions contre-transférentielles ${ }^{13}$ de bien des thérapeutes quand leurs patients expriment leur foi. C'est du moins l'opinion de Jonna Lannert ${ }^{14}$. Elle avance que la perception du phénomène religieux comme étant irrationnel et signe de troubles émotionnels indispose encore de nombreux thérapeutes quand le patient fait état de sa spiritualité ou de sa foi au cours du processus thérapeutique. La résistance du thérapeute à explorer ses propres conflits religieux non résolus peut resurgir dans une réaction contre-transférentielle qui nuirait au processus en discréditant la croyance religieuse exprimée par le patient, par exemple. L'impact de cela peut être très grand puisqu'il est démontré que les patients peuvent acquérir les valeurs spécifiques et les attitudes de leur thérapeute plutôt que des croyances personnelles matures, adaptées et intégrées. L'influence du thérapeute et le caractère très intime de la croyance religieuse militent en faveur d'une clarification

13. Le contre-transfert est ici défini comme les perceptions et les réactions distorsionnées du thérapeute face au patient sur la base de structures internalisées inconscientes (ZEIGER et LEWIS, "The Spirituality Responsible Therapist ", p. 420). Référer à la section 5.2.3 du présent article qui développe davantage ce thème du contre-transfert.

14. J. LANNERT, "Resistance and Countertransference Issues with Spiritual and Religious Patients ", Journal of Humanistic Psychology, 31 (1991) p. 68-76. 
par le thérapeute de ses propres résistances face au religieux pour éviter de les balayer sous le "tapis de la neutralité » et risquer ainsi qu'elles ressortent subtilement mais non moins perceptiblement pour le patient.

Le thérapeute peut aussi, par manque de familiarité avec le phénomène religieux, manquer une occasion d'aider le patient à explorer la signification et les conflits sous-tendus par sa pratique et par ses croyances religieuses. Toutefois, il est aussi vrai qu'un thérapeute croyant peut ignorer les enjeux qu'exprime le discours religieux de son patient par crainte que son option ne transparaisse dans ses interventions. Enfin, la confrontation avec des angoisses existentielles telles la maladie, le vieillissement et la mort peut inhiber le thérapeute et l'empêcher d'être à l'écoute de son patient et d'intervenir adéquatement.

Le préjugé négatif envers la question religieuse est fortement lié aux positions de Freud qui ont influencé nombre de psychanalystes et d'intervenants en santé mentale. Selon le psychanalyste américain Otto Kernberg ${ }^{15}$, il existait jusqu'à récemment une tendance à prendre une position philosophique athée chez les psychanalystes de renom. C'était dans la lignée de Freud qui affirmait qu'à long terme, un système rationnel de convictions morales et une éthique fondée sur la raison remplaceraient la religion organisée qui n'aurait alors plus à civiliser les rapports humains et à renforcer des interdits tout en procurant une consolation au besoin. Freud croyait qu'il serait possible d'abandonner l'idée de Dieu et de fonder la culture sur une base plus rationnelle d'attentes et d'interdits. L'intelligence, selon lui, offrait une alternative à la religion pour le contrôle des besoins pulsionnels.

Cette affirmation de Freud du triomphe de la rationalité étonne, puisqu'il avait tant insisté sur l'infiltration inévitable de motivations inconscientes (particulièrement agressives) dans la conscience et la raison. Cette affirmation semble aussi contredire ce qu'il a reconnu comme l'inévitable conflit entre les dominants et les plus démunis ainsi que les dangers inhérents à la psychologie des masses. Kernberg croit que la psychanalyse touche ici, avec Freud, les frontières qui devraient la distinguer de la philosophie : «Elle entre en zone dange-

15. O.F. KeRnBerG, "Psychoanalytic Perspectives on the Religious Experience ", American Journal of Psychotherapy, 54 (2000) p. 452-476. 
reuse et risque de devenir une Weltanschauung, contredisant ainsi l'esprit scientifique de l'exploration psychanalytique ${ }^{16}{ }^{\prime}$.

Sur ce sujet, Régis Debray, auteur de Dieu, un itinéraire, confiait ce qui suit à un journaliste de l'Express ${ }^{17}$ : « Le message des Lumières consistait à dire : la foi est un problème dont la science aura la solution. Autrement dit, plus il y aura de raison, moins il y aura de superstition, plus les écoles seront pleines, plus les lieux de culte seront vides... Or preuve est faite que non. Après le scientisme, religion de la science, on revient aux religions du Livre ou à des succédanés sectaires. » Tout comme Kernberg, il invite à distinguer l'espace du savoir, qui a son autonomie propre, de l'espace du sens.

Le triomphe de la rationalité annoncé par Freud (ainsi que par les Lumières) n'est pas seulement en contradiction avec ses propres découvertes, mais il est sérieusement mis en doute par l'expérience du XXe siècle. Pendant cette période deux systèmes idéologiques prétendant développer une rationalité et une moralité fondées dans l'analyse scientifique du développement ont mené aux pires atrocités, le nazisme et le communisme. Kernberg soutient donc qu'il importe, tout comme Freud l'a fait pour l'art, de reconnaître une existence autonome à un système universel de valeurs qui ne soient subordonnées ni à la raison, ni à la science $^{18}$. Les systèmes éthiques universels, comme ceux que produit la religion organisée, deviennent un rempart majeur et nécessaire contre les menaces de régressions majeures de groupe ${ }^{19}$.

\subsection{Le positionnement de l'intervenant face à la question religieuse : un continuum}

Il existe aux États-Unis depuis 1980 un mouvement qui plaide pour une plus forte intégration du religieux et de la spiritualité dans la psychothérapie alors qu'en Europe on ne soulève pas cette question dans les milieux de la psychologie ${ }^{20}$. L'intervenant doit donc pouvoir se situer

16. KeRnBerg, "Psychoanalytic Perspectives », p. 455.

17. R. Debray, "Pourquoi Dieu est de retour. Islam, christianisme, judaïsme - propos recueillis par Christian Makarian ", L'Express, 2626 (1er novembre 2001), p. 44.

18. DEBRAY, "Pourquoi Dieu est de retour », p. 456.

19. DEBRAY, "Pourquoi Dieu est de retour », p. 472. 
sur un continuum qui va d'un pôle où domine la vision religieuse à un autre où elle est totalement exclue. À un extrême, on affirme que l'institution religieuse devrait rapatrier l'intervention thérapeutique et «faire de la psychologie la servante de l'Église ${ }^{21}$ ». À l'autre extrême, on trouve des thérapeutes qui ont un préjugé défavorable à toute croyance religieuse et qui le manifestent consciemment ou non à leur patient. À bonne distance de ces radicalismes se retrouvent bien des thérapeutes qui font une place importante à la spiritualité dans leur intervention en allant jusqu'à dévoiler leurs croyances et à proposer des voies de guérison enracinées dans ces croyances. D'autres insistent pour distinguer les systèmes de croyance religieuse des processus psychologiques $^{22}$. Ils considèrent tous les discours religieux sous l'angle des enjeux psychologiques qu'ils sous-tendent et à la lumière de critères cliniques. Entre ces deux pôles, on trouve des thérapeutes qui se reconnaissent davantage dans la "neutralité bienveillante » de Freud où il ne s'agit pas de se désintéresser du patient, mais de s'abstenir de le guider selon les valeurs spirituelles du thérapeute ${ }^{23}$.

\subsubsection{Pour une intégration des besoins spirituels}

avec les besoins psychologiques

Il en est, tel le psychologue américain Bergin ${ }^{24}$, qui plaident pour une évaluation spirituelle du patient et une incorporation des valeurs religieuses dans la psychothérapie. Selon lui, le thérapeute est de toute façon un prédicateur séculier lorsqu'il tente d'influencer son patient dans le sens d'un comportement acceptable socialement et il vaut mieux l'influencer avec les valeurs supérieures que sont les valeurs religieuses ${ }^{25}$. D'autres prônent l'exploration de l'aspect religieux dans la vie du patient pour aider le thérapeute à mieux comprendre celui-

20. J. CORVELEYN, «In defense of Benevolent Neutrality. Against a Spiritual Strategy ", The Journal of Individual Psychology, 56 (2000) p. 343-352.

21. D.E. FITCH, « The Need for more Preaching in the Psychologist's Office or why Therapy never Should Have Left the Church in the First Place ", Pastoral Psychology, 48 (2000) p. 197-209.

22. ZEIGER et LEWIS, "The Spirituality Responsible Therapist ", p. 416.

23. CORVELEYN, "In Defense of Benevolent Neutrality ».

24. A.E. BERGIN, "Psychotherapy and Religious Values ", Journal of Consulting and Clinical Psychology, 48 (1980) p. 95-105.

25. ZEIGER et LEWIS, "The Spirituality Responsible Therapist ", p. 416. 
ci et aider le patient à mieux saisir le lien entre des aspects problématiques de sa vie et un possible conflit religieux en lui-même ${ }^{26}$. À travers l'exploration religieuse, le thérapeute peut aussi reconnaître chez le patient un besoin d'intégrer des aspects religieux et psychologiques de lui-même pour être en meilleur santé psychologique ${ }^{27}$.

Pour Hemert, qui prône une formation aux questions religieuses chez les futurs travailleurs sociaux, la meilleure façon de se prémunir d'un effet négatif subtil mais réel des croyances religieuses sur le processus thérapeutique est d'ouvrir cette question d'emblée avec le patient ${ }^{28}$. Selon elle, le silence sur la question religieuse, son exclusion tacite du cadre, aurait un effet répressif. Elle suggère donc que les travailleurs sociaux parlent ouvertement de la religion avec leur patient plutôt que de risquer d'avoir une pratique discriminatoire en ne respectant pas la différence de celui-ci. Hemert approuve ceux qui refusent de soutenir une religiosité rigide, infantile et autodestructrice mais elle craint d'offrir un service inadéquat et non éthique en niant au travail social toute dimension religieuse. Selon elle, la dimension religieuse est une dimension centrale de la vie du patient; ce dernier devrait donc se sentir libre de l'aborder dans la démarche thérapeutique. Plus loin, elle qualifie la religion d'incontournable si l'on vise à favoriser l'autodétermination du patient car elle est une préférence personnelle, une dimension de la personnalité et une condition sociale de la personne. Hemert doute que l'intervenant puisse être au service de ses patients, maintenir la primauté de leur intérêt, tout en excluant cette dimension centrale de leur vie ${ }^{29}$.

\subsubsection{Pour une distinction entre les domaines religieux et psychothérapiques ${ }^{30}$}

Clark, un travailleur social américain, met lui aussi de l'avant l'autodétermination du patient en affirmant le besoin de protéger l'intervention thé-

26. J. GIGLIO, « The Impact of Patients' and Therapists' Religious Values on Psychotherapy ", Hospital and Community Psychiatry, 44 (1993) p. 768-772. 27. ZEIGER et LEWIS, "The Spirituality Responsible Therapist ", p. 417.

28. K. Amato-VON HemerT, "Should Social Work Education Address Religious Issues ? Yes! ", Journal of Social Work Education, 30 (1994) p. 7-11. 29. AMATO-VONHEMERT, «ShouldSocialWorkEducation », p.8.

30. C'est à partir de l'opinion de Clark, un travailleur social qui prenait part à un débat autour de l'introduction ou non de la dimension religieuse dans la formation des étudiants que nous aborderons cette position de l'intervenant. 
rapeutique de l'introduction de la dimension religieuse par l'intervenant ${ }^{31}$. Il redoute l'aspect contraignant de la religion et veut préserver le patient des codes moraux des Églises et de tout ce qu'elles pourraient lui imposer $^{32}$. Il soupçonne même une visée apologétique chez certains intervenants qui voudraient reconnaître au travail social une dimension religieuse. Il propose plutôt de reconnaître la croyance religieuse du patient comme une des dimensions de sa personnalité que l'intervenant se doit de respecter comme il le fait pour les autres dimensions.

Clark distingue l'intervention sociale du travail de counseling pastoral et donne prééminence aux critères cliniques par rapport aux critères religieux. De même, il juge risquée l'utilisation de techniques religieuses (prière, rituels) dans le contexte thérapeutique à moins d'une très bonne connaissance de la culture et de la religion. Selon lui, l'exclusion de l'utilisation du religieux dans l'intervention protège davantage le patient qu'elle ne lui nuit. "La sécularisation garantit à chacun le droit à ses croyances et c'est une protection essentielle du patient que d'exclure le religieux de toute intervention professionnelle, à moins qu'il ne vienne de celui-ci, écrit-il ${ }^{33}$ ».

Selon Zeiger et Lewis ${ }^{34}$, l'évaluation et l'exploration religieuse ne sont pas du domaine de la psychothérapie, à moins que, d'un commun accord, le patient et le thérapeute en décident autrement et s'entendent sur un cadre qui inclut explicitement la dimension religieuse. Selon eux, l'abstinence du thérapeute à communiquer implicitement ou explicitement ses croyances religieuses peut avoir un impact positif sur la psychothérapie. D'abord il préserve le cours de la thérapie et la dynamique transférentielle de l'influence de la question religieuse et ensuite il écarte l'impact d'une similarité ou d'une différence de croyance entre le thérapeute et le patient ${ }^{35}$. Les prochains paragraphes relèvent des résistances, décrites par Zeiger et Lewis, qui peuvent surgir autour de la question religieuse dans la psychothérapie.

31. J. ClarK, "Should Social Work Education Address Religious Issues ?

No! ", Journal of Social Work Education, 30 (1994) p. 11-15.

32. CLARK, "Should Social Work Education ", p. 15.

33. CLARK, "Should Social Work Education ", p. 15.

34. ZEIGER et LEWIS, "The Spirituality Responsible Therapist ", p. 423.

35. ZEIGER et LEWIS, "The Spirituality Responsible Therapist ", p. 423. 
D'abord la perception qu'a le patient du thérapeute peut être altérée (transfert) à partir de la vision qu'a le patient de la divinité. Tout comme les images internalisées des parents peuvent être projetées sur le thérapeute, le matériel religieux peut aussi générer le déplacement de l'image internalisée de la divinité sur le thérapeute. Celui-ci devient alors suridéalisé, surestimé et vu comme omniscient et omnipotent à la manière de la perception qu'a le patient de la divinité ${ }^{36}$.

Il peut aussi arriver que le patient place le thérapeute dans un double rôle tentant ainsi de se relier à lui davantage comme à un pair sur le plan religieux qu'à un thérapeute. Ceci se produit spécialement quand le patient sent que le thérapeute a des croyances religieuses similaires aux siennes. Le patient peut alors être tenté de contourner un nécessaire travail thérapeutique en s'alliant au thérapeute sur le plan religieux ${ }^{37}$. Ça peut être le cas quand un patient s'adresse à un psychologue dont il sait qu'il est aussi prêtre. Un collègue qui est dans cette situation me confiait qu'il avait dû occasionnellement clarifier son rôle auprès de patients qui lui demandaient l'absolution et qui étaient pourtant venus le consulter comme psychologue.

Il arrive aussi que le patient refuse de considérer sa croyance religieuse d'une façon personnelle en l'abordant uniquement d'une façon convenue et intellectuelle, évitant ainsi toute responsabilité personnelle pour ses croyances. Le patient peut aussi inconsciemment inclure le thérapeute dans le giron de ses croyances et lui rendre très inconfortable toute exploration qui sortirait des balises conventionnelles et idéologiques qu'il lui a fixées.

\subsubsection{La neutralité bienveillante ${ }^{38}$}

L'attitude de neutralité bienveillante du thérapeute se rapproche de la précédente. Elle s'en distingue toutefois par une attitude moins " militante " et plus « neutre » face à la distinction des domaines religieux et thérapeutiques. Elle est présentée par Corveleyn ${ }^{39}$ qui propose de revoir le concept de neutralité chez Freud sans se laisser prendre par l'athéisme affirmé de celui-ci. Selon lui, la neutralité prô-

36. ZEIGER et LEWIS, "The Spirituality Responsible Therapist ", p. 419.

37. ZeIGER et LEWIS, "The Spirituality Responsible Therapist ", p. 419.

38. CORVELEYN, "In Defense of Benevolent Neutrality », p. 343-352.

39. CORVElEYN, «In Defense of Benevolent Neutrality », p. 344. 
née par Freud n'est pas de l'indifférence, mais une attitude de sympathie envers le patient. Elle vise à laisser au patient le plus de liberté possible pour qu'il redécouvre pour lui-même la façon dont il se situe par rapport aux questions importantes de la vie.

L'auteur écrit comment le thérapeute n'est pas un agent de pastorale dont la tâche serait d'engager activement la conversation sur des thèmes liés à la foi (croyances, attitudes, émotions) avec des patients qui consultent par rapport à toutes sortes de problèmes existentiels. Mais le thérapeute ne doit pas non plus avoir trop de retenue quant il s'agit de diriger la conversation dans le domaine religieux ou spirituel. Car le tact du thérapeute manifesté par un silence alors qu'une intervention supportante ou exploratoire serait de mise, risquerait alors de devenir un alibi pour agir sa résistance personnelle face au religieux. Il est déjà très difficile pour un patient d'aborder les thèmes religieux ou spirituels dans une société sécularisée. Si la résistance du thérapeute s'ajoute à cela, la difficulté s'accroît.

Il est arrivé que des analystes ont transposé au plan thérapeutique l'explication freudienne de la religion comme phénomène culturel, visant donc à déconstruire la croyance religieuse, comme le fait la psychanalyse avec toute illusion potentiellement inhibitrice du développement du patient ${ }^{40}$. À son ami et pasteur protestant Oskar Pfister, Freud écrivait : « La psychanalyse n'est ni religieuse, ni non religieuse, mais un instrument sans parti que le prêtre et le laïc peuvent utiliser au service de la personne souffrante ${ }^{41} »$. Ailleurs il lui écrivait ceci : "Comme pasteur, vous avez le droit d'en appeler à tous les renforcements à votre disposition, mais comme analystes, nous devons être plus réservés, et nous devons mettre l'accent sur l'effort pour rendre le patient indépendant ${ }^{42}$ ».

Le psychanalyste vise donc à ce que le patient obtienne une plus grande liberté face à des inhibitions et déformations internes ${ }^{43}$. Cette

40. CORVEleyN, "In Defense of Benevolent Neutrality », p. 347.

41. Sigmund FREUD et Oskar PFISTER, Psychoanalysis and Faith. The Letters of Sigmund Freud and Oskar Pfister / édité par H. MENG et E.L. FreuD, trad. par E. Mosbacher, New York, Basic Books, 1963 (allemand 1963), p. 17. Traduction française par l'auteur.

42. FREUD et PFISTER, Psychoanalysis and Faith, p. 113.

43. CORVELEYN, "In Defense of Benevolent Neutrality », p. 348. 
action libératrice peut promouvoir le développement d'une expérience religieuse personnelle, ou elle peut libérer la personne de représentations ou de pratiques religieuses oppressives. Mais cette libération pour ou de la religion n'est pas l'objectif ni de la psychanalyse, ni de la psychothérapie en général.

Selon Corveleyn, la psychothérapie peut aider quelqu'un à se libérer pour faire de la place à un désir religieux découvert en lui. Mais le psychothérapeute ne devrait pas nuire à la découverte spirituelle du patient (croyant ou non) en lui indiquant une voie vers une spiritualité ou une religion préfabriquée. À cause de la force du transfert, indiquer une voie spirituelle à un patient, à partir des choix du thérapeute plutôt qu'à partir du patient, peut compromettre l'intégration d'une spiritualité personnelle dans le processus thérapeutique ${ }^{44}$.

La neutralité signifie que le thérapeute ne se place pas dans une position où il évaluerait les valeurs religieuses, éthiques ou socio-politiques du patient ainsi que les comportements qui y seraient liés. Une telle neutralité doit prévaloir quant au contre-transfert du thérapeute. Bien sûr, celui-ci n'est pas de glace, mais il doit s'assurer qu'il ne se met pas dans le chemin du processus thérapeutique du patient en laissant s'immiscer ses affects personnels dans la thérapie ${ }^{45}$.

Corveleyn conclut en répétant comment le thérapeute ne doit pas guider son patient vers l'adhésion à des valeurs qui seraient les siennes. En même temps, la neutralité bienveillante n'est pas de la passivité ; au contraire, le thérapeute doit soutenir le patient, l'encourager et le stimuler à habiter sa liberté. Si surviennent des thèmes religieux, le rôle du thérapeute sera d'aider le patient à les explorer en surmontant sa timidité ou ses craintes par rapport à de tels thèmes ${ }^{46}$.

Kernberg abonde dans ce sens. Cliniquement, écrit-il, une des fonctions de la psychanalyse est d'explorer dans quelle mesure la religiosité, comme désir mature pour un système de moralité transpersonnel et de valeurs éthiques, est disponible à nos patients. La fonction du psychanalyste n'est pas celle de conseiller pastoral ou de guide vers un tel système de valeurs universelles. La fonction du psy-

44. CORVELEYN, "In Defense of Benevolent Neutrality », p. 348.

45. CORVELEYN, "In Defense of Benevolent Neutrality ", p. 349.

46. CORVELEYN, "In Defense of Benevolent Neutrality ", p. 350. 
chanalyste est plutôt de libérer le patient de conflits inconscients qui limitent cette capacité. Cela peut impliquer la confrontation systématique ainsi que l'exploration et la résolution de conflits inconscients qui empêchent le développement du souci pour l'autre, de la culpabilité, de la réparation, du pardon, de la responsabilité et de la justice comme des aspirations fondamentales de l'individu. La psychanalyse doit aussi aider certains patients à se libérer d'un usage de la religion formelle comme rationalisation de la haine et de la destructivité dirigée contre soi et les autres ${ }^{47}$.

\subsection{Qúen penser?}

Paradoxalement, les thérapeutes qui se réclament des trois positions le font au nom du même respect de l'autodétermination du patient. Les premiers défendent leurs patients au nom d'une ouverture sur la dimension religieuse qu'ils estiment centrale dans la vie de ceux-ci, les deuxièmes veulent préserver le développement de leurs patients des éléments plus contraignants associés à la religion, et les derniers adoptent une attitude de neutralité bienveillante pour laisser à leurs patients un espace où peut émerger ou non une croyance religieuse.

C'est cette dernière position que je privilégie en même temps que je juge nécessaire de rester ouvert aux préoccupations des deux autres. Avec des patients aux croyances religieuses plutôt conservatrices (par exemple, Juifs orthodoxes, chrétiens fondamentalistes, Témoins de Jéhovah, etc.), il peut être nécessaire d'ouvrir d'emblée la question religieuse et de rassurer le patient sur le fait que la thérapie n'exigera pas de lui qu'il croit en quoi que ce soit dont il n'est pas convaincu ${ }^{48}$. Pour les patients qui accordent de l'importance à la dimension religieuse, plusieurs suggèrent que l'incorporation de perspectives spirituelles dans le traitement psychothérapique séculier peut contribuer à l'efficacité de celui-ci ${ }^{49}$. Il reste à voir selon quelles modalités cela pourrait être fait. Pour d'autres patients, l'intégration des aspects religieux et psychologique peut être un objectif de la thérapie ${ }^{50}$. Cela

47. KernberG, "Psychoanalytic Perspectives », p. 475.

48. ZEIGER et LEWIS, "The Spirituality Responsible Therapist ", p. 418.

49. W.R. MiLler et C.E. THORESEN, "Spirituality and Health" dans W.R. MILLER, dir., Integrating Spirituality into Treatment. Resources for Practitioners, Washington, DC, American Psychological Association, 1999, p. 14. 
demande du thérapeute qu'il soit ouvert à discuter du contenu religieux pour la croissance du patient ${ }^{51}$. Comme on l'a dit ci-haut, la dimension religieuse peut être davantage défensive et signe d'une résistance mais elle peut être aussi le lieu de la manifestation et de l'exacerbation d'une pathologie. Dans ce dernier cas, il devient essentiel d'appliquer les critères cliniques au matériel religieux.

En même temps que je privilégie la neutralité bienveillante, il reste que le thérapeute peut afficher une fausse neutralité et faire ainsi plus de dommages que de bien en voulant préserver l'espace thérapeutique de toute influence du religieux. Ainsi un thérapeute qui garde le silence sous prétexte de respect et de tact peut subtilement discréditer la croyance exprimée par un patient et évacuer ainsi toute possibilité pour celui-ci d'aborder cet aspect de sa vie dans le cadre de la thérapie.

Au-delà des positions adoptées par le thérapeute, c'est d'abord la prise de conscience de ce positionnement qui est essentiel. Où est-ce que je me situe par rapport à la foi religieuse, à la spiritualité ? Quelles sont mes réactions à l'expression par un patient de préoccupations spirituelles ? Il s'agit donc moins de connaissances à parfaire par rapport au domaine de la religion et de la spiritualité que d'une prise de conscience de ses propres positions autour de ces questions. Le thérapeute doit savoir comment rester subjectivement détaché, séparant ses valeurs et intérêts personnels de ceux du patient. Il est bien connu que des thèmes reliés à la problématique du patient ainsi qu'à celle du thérapeute posent un défi particulier pour une psychothérapie efficace ${ }^{52}$. Des thérapeutes vont parfois refuser des patients qui les renverraient à des questions auxquelles ils sont particulièrement sensibles.

\section{Diverses situations d'émergence des dimensions religieuse et spirituelle dans le contexte d'intervention}

La dimension religieuse peut surgir dans différentes situations qui exigent diverses approches de la part de l'intervenant. Ainsi, l'intervenant

50. M.H. SPERo, Psychotherapy of the Religious Patient, Northvale, N.J., Jason Aronson, 1995.

51. L.H. Henning et F.J. TiRrell, "Counselor Resistance to Spiritual Exploration ", Personnel and Guidance Journal, 61 (1982) p. 92-95.

52. ZEIGER et LEWIS, "The Spirituality Responsible Therapist ", p. 418. 
T A B LE A U 1

Situations d'émergence de la dimension religieuse ou spirituelle dans l'intervention psychosociale

\begin{tabular}{|c|c|c|}
\hline \multicolumn{2}{|c|}{ Religieux intimement lié au culturel } & \multirow{2}{*}{\begin{tabular}{|l|}
$\begin{array}{l}\text { Religieux dissocié de la culture } \\
\text { d'origine }\end{array}$ \\
$\begin{array}{l}\text { C) Adhésion religieuse adaptative } \\
\text { (convertis) }\end{array}$ \\
\end{tabular}} \\
\hline A) Immigrants & $\begin{array}{l}\text { B) Québécois de } \\
\text { souche, catholiques } \\
\text { ou protestants }\end{array}$ & \\
\hline
\end{tabular}

issu d'un milieu catholique ne se comporte pas de la même façon avec un converti à une religion étrangère à sa culture qu'avec une personne âgée pratiquante dominicale assidue. Dans le Tableau 1, j'esquisse différentes situations dans lesquelles peut émerger la dimension religieuse. D'abord, il y a celle des immigrants qui pratiquent leur religion (A-D) et dont on juge qu'elle est un mécanisme adaptatif utile et même essentiel à l'équilibre et à l'identité, spécialement dans une situation de $c h o c$ migratoire. En deuxième lieu, il y a le Québécois de souche qui garde un lien vivant ou qui a renoué avec la foi de son enfance (B-D). Cette situation exige de l'intervenant qu'il soit au clair avec le rapport qu'il entretient avec cette religion. Le troisième cas est celui d'une personne ayant adhéré à une religion qui n'est pas celle de son enfance ou de sa culture d'origine $(\mathrm{C}-\mathrm{D})$ et qui y puise des ressources qui l'aident à vivre et à s'adapter. Enfin, pour toutes ces situations, il est possible que le clinicien juge que la croyance religieuse ou la spiritualité de la personne ait un impact négatif sur la personne (A-E, B-E, C-E).

\subsection{Accueil du religieux de l'autre immigrant}

L'immigration constitue une voie par laquelle nous arrive des conceptions du religieux souvent très différentes des nôtres. Ainsi, si l'on tient compte des vagues d'immigration récentes, le contact avec un musulman ou un chrétien baptiste peut heurter notre conception très séculière des croyances religieuses qui confine celles-ci au domaine strictement privé, alors qu'elles font partie intégrante de la vie pour les gens de ces cultures. Une telle différence vaut aussi pour des communautés minoritaires implantées au Canada depuis plusieurs décennies, telles les Juifs ; par exemple, il est inconcevable pour un Juif ortho- 
doxe de consulter un thérapeute sans la présence ou à tout le moins l'autorisation du rabbin. À cause de cette intrication de la vie et de la religion, Altareb invite l'intervenant à évaluer l'identité religieuse et la religiosité du patient musulman pour cerner l'importance qu'il accorde à la religion ${ }^{53}$. Celui pour qui les valeurs de l'islam sont très importantes et guident sa vie a peut-être besoin d'être rassuré que le thérapeute tentera de comprendre sa perspective sans tenter de la changer. Le thérapeute peut aussi faire sentir au patient, quand surviennent les questions religieuses, que ses valeurs religieuses sont acceptées dans le processus thérapeutique et qu'elles font partie de la solution à ses problèmes. Altareb va jusqu'à proposer au thérapeute de se renseigner sur le point de vue islamique d'une question particulière surgie en thérapie, par exemple l'origine coranique ou non de l'interdiction posée à la femme de travailler à l'extérieur ${ }^{54}$. Toutefois, il faut dire qu'il y a peu de chance qu'un patient qui s'identifie totalement à la religion et aux valeurs musulmanes se présente en thérapie. Il ira plutôt chercher de l'éclairage d'un imam ou d'un guérisseur ${ }^{55}$ qui considérera sa situation d'un point de vue religieux, islamique.

Il arrive souvent que l'aspect religieux de l'immigrant soit perçu par l'intervenant comme archaïque, porteur de conceptions dépassées, contraignantes et peut-être même injustes. Ainsi, la soumission de la patiente musulmane à son mari peut heurter et désorienter le thérapeute. Carlo Sterlin décrit en trois phases le cheminement d'atteinte de la "maturité professionnelle interethnique ${ }^{56}$ ». D'abord il parle de la fuite de la pratique vers les homo-ethniques, car avec les hétéro-ethniques l'intervenant n'y comprend rien et est irrité par le manque de ponctualité, la communication circonstancielle, l'aura paranoïaque des interactions, l'envahissement de son espace et de son temps, l'ambiva-

53. B.Y. Altareb, "Islamic Spirituality in America. A Middle Path to Unity ", Counseling and Values, 41 (1996) p. 29-38.

54. AlTAREB, "Islamic Spirituality in America ".

55. A. AL-KRENAWI et J.R. GrahAM, "Social Work and Koranic Mental Health Healers ", International Social Work, 42 (1999) p. 53-65

56. C. STERLIN, "La référence culturelle dans une pratique psychiatrique en milieu haïtien à Montréal » dans H. CORIN, S. LAMARRE, P. MignEAULT et M. TOUSIGNANT, dir., Compte-rendu du colloque : Regards anthropologiques en psychiatrie, Ed. Girame, 1987, pp. 97-109. L'article a été consulté sous sa forme manuscrite. 
lence relative à la langue de communication, la transgression de la frontière professionnelle/privée, les attentes de divination et de magie, etc. Dans un deuxième temps, l'intervenant applique des outils occidentaux à la compréhension de la réalité ethnique. Il est tenté d'imposer ses schèmes d'interprétation et ses outils d'intervention à une réalité rebelle qui lui échappe de toutes parts. Pour retrouver une impression de maitrise, il peut développer une "psychologie du commandeur " se traduisant par une attitude de mépris et une rigidité sans nuances dans les interventions. Enfin, Sterlin parle de la recherche d'une perspective authentiquement transculturelle. Il s'agit à la fois d'une démarche intellectuelle et d'un processus de maturation personnelle. "Car le défi, écrit-il, est non seulement de transcender les schémas officiels pour considérer la pathologie et la psychiatrie en tant que faits de culture, mais de faire un cheminement personnel nous permettant de nous situer dans la dynamique sociale et politique globale et de totaliser nos contradictions personnelles ${ }^{57}$ ». Il va sans dire que ceci vaut autant pour le champ multiculturel que pour la diversité religieuse.

\subsection{Accueil du religieux de l'autre qui conserve un lien avec la religion de son enfance}

On pourrait ici parler du religieux qui est similaire à celui de l'intervenant et qui risque donc de susciter une réaction contre-transférentielle chez celui-ci. Le contre-transfert peut être compris comme les perceptions et les réactions distorsionnées du thérapeute face au patient sur la base de structures internalisées inconscientes ${ }^{58}$. Des réactions contre-transférentielles négatives peuvent survenir quand des croyances religieuses apparemment similaires ont des origines ou des fonctions différentes pour le patient et le thérapeute, ou lorsque le thérapeute s'identifie démesurément aux conflits religieux du patient. La difficulté à tolérer le questionnement religieux d'un patient, une forte réaction émotionnelle aux luttes d'un patient, jugeant celui-ci irrationnel, et des fantaisies intenses de sauveur sont autant de manifestations possibles de contre-transfert chez le thérapeute religieux ${ }^{59}$.

57. STERLIN, « La référence culturelle».

58. ZeIGER et LEWIS, « The Spirituality Responsible Therapist », p. 420.

59. M.H. SPERO, « Countertransference in Religious Therapists of Religious Patients ", American Journal of Psychotherapy, 35 (1981) p. 565-576. 
Le cas de l'identification démesurée aux conflits religieux du patient est la forme de contre-transfert qui suscite la plus forte résistance à l'exploration religieuse et au travail des difficultés qui lui sont reliées. Souvent l'exploration religieuse du patient va provoquer de l'anxiété chez le thérapeute ainsi qu'une peur intense de questionner ses propres croyances. Les conflits personnels du thérapeute et les défenses non analysées peuvent mener à l'évitement du matériel religieux par le biais d'interprétations trop rapides, par un changement de sujet sans exploration suffisante, par des débats philosophiques sans visées thérapeutiques, ou par le discrédit des contenus religieux dans la thérapie en l'interprétant comme une résistance du patient alors qu'une telle désignation peut très bien être le signe d'une résistance du thérapeute. Souvent, le thérapeute justifiera l'évitement des sujets religieux par la projection sur le patient de sa propre incapacité à tolérer l'anxiété liée à l'exploration de thèmes religieux.

\subsection{Accueil du religieux de l'autre converti}

Au Québec bien des catholiques se sont convertis à une religion orientale telle le bouddhisme ou à une Église chrétienne fondamentaliste ou encore à une secte. Nous nous attarderons ici à la conversion à des Églises chrétiennes plus sectaires. Plusieurs auteurs comparent l'adhésion à une secte et la dépendance qui s'ensuit à la dépendance à des substances. Ainsi, Abgrall fait s'équivaloir des critères du trouble addictif à la dépendance sectaire tels l'impossibilité de résister aux impulsions à réaliser un type de comportement, la sensation croissante de tension précédant immédiatement le début du comportement, le plaisir ou le soulagement pendant sa durée ${ }^{60}$, etc. Une telle analogie est qualifiée d'analogie de surface par Pedinielli qui s'interroge sur la légitimité de la transposition de concepts et de modèles psychopathologiques dans le domaine de la religion ${ }^{61}$. On omet alors des éléments psychologiques impliqués dans l'engagement religieux et la croyance. Il en nomme trois : le lien avec le gourou, l'effet structurant de l'appartenance au mouvement religieux fondamentaliste et la

60. J.-M. AbGRALL, La mécanique des sectes, Paris, Payot, 1996, p. 290-293. 61. J.-L. PEDINIELli, "Sectes et addictions", présenté au colloque Manipulations mentales, Centre d'études des nouvelles religions, Paris, 1997, p. 36-45. 
quête de sens qui y est poursuivie et soutenue. En lien avec ces observations, Denton, dans un article sur l'évaluation et le traitement de la famille appartenant à un mouvement fondamentaliste, attire l'attention de l'intervenant sur les apports du fondamentalisme à une famille par rapport à la socialisation, le sentiment d'appartenance ${ }^{62}$, etc. Il réfère à des études ${ }^{63}$ qui montrent comment le fondamentalisme peut servir à instaurer des frontières dans un monde qui peut menacer des croyances centrales pour la famille. Il invite donc l'intervenant à une approche qui ne soit pas confrontante mais qui considère l'aspect adaptatif du fondamentalisme religieux.

\subsection{Le normal, le patbologique et le thérapeutique}

Il est reconnu que l'engagement dans une religion est souvent inversement relié aux troubles physiques, mentaux et d'abus de substances. Mais si l'engagement religieux est un facteur protecteur et vitalisant, pourquoi existe-t-il des personnes pour qui il ne joue pas ce rôle? Gorsuch et Miller reconnaissent qu'il existe peu de recherche sur ce sujet mais proposent trois hypothèses ${ }^{64}$ :

1. Leur religion est protectrice mais la personne est tout de même débordée par d'autres influences.

2. Leur religion a un effet négatif, par exemple si elle restreint la divinité à une figure autoritaire et jugeante.

3. Les croyances ou comportements religieux de la personne dérivent du trouble de la personne (par exemple, l'autocritique dans la dépression clinique). Ainsi, le traitement approprié peut redonner à la croyance religieuse une forme plus saine.

La question pour l'intervenant est donc de savoir comment discerner les cas où la religion a un effet négatif. Quand le religieux du patient devient-il nuisible et destructeur, quand est-il une fuite adaptative à

62. R.T. DENTON, "The religiously Fundamentalist Family. Training for Assessment and Treatment ", Journal of Social Work Education, 26 (1990) p. 6-14.

63. F. M. Ethridge et J. R. Feagin, "Varieties of Fundamentalism. A Conceptual and Empirical Analysis of Two Protestant denominations ", Sociological Quarterly, 20 (1979) p. 37-48.

64. R.L. GORSUCH et W.R. MilleR, "Assessing Spirituality » dans MilleR, Integrating Spirituality into Treatment, p. 48. 
respecter ? Vergote tente d'élaborer des critères de normalité et de pathologie du religieux ${ }^{65}$. Il suggère, par exemple, que si la quête religieuse absorbe à ce point l'homme qu'il n'est plus en mesure de se libérer pour le travail nécessaire, alors il faut suspecter pareil engagement religieux.

En même temps qu'il est nécessaire d'être alerté par rapport à la pathologie que peut sous-tendre l'engagement religieux, il est tout aussi important de garder à l'esprit les aspects thérapeutiques de la croyance religieuse. Les études de psychologie de la religion indiquent quatre sphères dans lesquelles les gens font appel à l'expérience de leurs croyances dans leur vie ${ }^{66}$. D'abord la religion peut être un mécanisme d'adaptation (coping) et permettre à des personnes de trouver du réconfort et du support dans des temps difficiles. Deuxièmement, la religion peut proposer aux gens des modèles auxquels ils peuvent s'identifier lors d'une épreuve. Ainsi, on peut privilégier un modèle ou un autre à cause du courage de l'un, de la persévérance de l'autre, etc. Dans le christianisme, le Christ est évidemment la figure d'identification par excellence, mais il y a aussi les saints. Troisièmement, la religion offre une façon d'interpréter le sens de la vie, le sens d'événements apparemment absurdes, qui peut alléger bien des angoisses à propos de la vie et de la mort. Ainsi, cette dame qui unira ses souffrances à celles du Christ, les offrant pour ceux et celles qu'elle aime. Ce jeune père sortant apaisé d'une rencontre de baptême parce qu'il a enfin des réponses à ses questions. Enfin, le soutien offert par des communautés croyantes n'est pas négligeable. Ainsi, plusieurs communautés offrent des ressources qui prennent la forme de services de pastorale, de communauté de support et même de services sociaux offerts aux membres.

Garder à l'esprit les aspects thérapeutiques du religieux, de même que ses aspects défensifs et même pathologiques, permet à l'intervenant de nuancer ses interventions selon ce qui survient. Ainsi le religieux de l'autre peut être perçu par l'intervenant comme un support, même si l'intervenant ne partage pas la croyance du patient. C'était le cas d'un

65. A. Vergote, Dette et désir. Deux axes chrétiens et la dérive pathologique, Paris, Seuil, 1978, p. 33.

66. J.W. Ellor, F.E. NetTing et J.M. Thibault, Religious and Spiritual Aspects of Human Service Practice, Columbia, SC, University of South Carolina Press, 1999, p. 70. 
thérapeute qui constatait l'effet bénéfique qu'avait la lecture de la Bible sur un patient anxieux et déprimé. Il a consciemment soutenu cette pratique malgré l'irritation qu'elle créait chez le conjoint du patient.

Dans une autre situation, le thérapeute a su déceler les aspects défensifs du religieux pour arriver à travailler les enjeux sous-jacents. C'est Spero qui rapporte le cas d'une patiente juive orthodoxe qui, en fin de séance, lui a demandé de lui accorder le pardon (mehilah) pour les injures qu'elle lui avait adressées durant la séance ${ }^{67}$. Ce pardon faisait partie d'un rituel de préparation au Yom Kippur et la patiente semblait lui accorder une importance cruciale pour mieux vivre la fête religieuse qui approchait. Spero, qui est juif pratiquant, aurait pu le lui donner comme un apaisement. Il aurait été fidèle à sa propre foi, attentif à la détresse de sa patiente et il aurait pu clore la séance sans bavure. Mais il a préféré explorer le sens de cette demande avec la patiente. Celle-ci a alors réalisé que derrière sa demande de pardon, il y avait sa colère contre son père, de qui elle aurait tant voulu recevoir le pardon. Mais ce dernier la laissait dans sa culpabilité et dans le doute quant à son amour pour elle : " Il y a encore de la colère et de la tristesse face à mon père. Obtenir son mehilah à travers vous n'est peut-être pas ce qu'il y a à faire ${ }^{68}$. " On peut voir ici comment le thérapeute a réussi à aller au-delà de la demande de la patiente sans pour autant mépriser le contenu religieux du discours ou se sentir contraint par l'autorité que peut donner à la demande sa teneur religieuse.

Enfin, Corin observe comment la dimension religieuse peut être vécue de façons diamétralement opposées par des personnes psychotiques. Elle remarque que :

le rapport au religieux de personnes psychotiques qui n'ont pas été réhospitalisées passe rarement par des structures institutionnelles traditionnelles. On peut dire qu'il se déploie davantage à leur marge, dans un espace protégé et toujours singulier. Ainsi plusieurs personnes ont établi une claire distinction entre des périodes de leur vie où elles ont été intensément impliquées dans une Église ou dans des rites religieux, sur le mode d'un " en trop " ou d'un excès; une telle implication est décrite comme ayant eu sur elles un effet d'engouffrement, comme ayant préci-

67. M.H. SPERO, "Transference as a Religious Phenomenon in Psychotherapy ", Journal of Religion and Health, 24 (1985) p. 8-25. 68. SPERO, "Transference as a Religious Phenomenon ", p. 20. 
pité la crise ou une rechute : J'étais tombée dans la religion. Par contraste, elles mentionnent aussi une autre modalité de rapport au religieux dont elles soulignent l'importance et le caractère bénéfique pour elles. On y retrouve l'empreinte de la position de retrait positif... Les personnes préfèrent alors souvent parler du sentiment d'une présence que d'une croyance claire en un Dieu déterminé, une présence qui semble leur permettre de se sentir accompagnées dans leur retrait intérieur, d'y reconstruire une certaine cohérence personnelle ${ }^{69}$.

On voit donc que, pour une personne psychotique, l'aspect religieux peut être ou bien thérapeutique ou bien pathologique selon que l'aspect contraignant du religieux est plus ou moins marqué à travers l'engagement institutionnel et la détermination du visage de Dieu. Ici encore, on remarque le besoin de discernement de la part du thérapeute. Par exemple, une famille en besoin de structure et de limites optera pour un engagement religieux institutionnel qui pourrait lui être bénéfique mais qui risquerait d'être nuisible pour une personne psychotique.

\section{Conclusion}

Dans cet article, j'ai voulu élargir la question religieuse au-delà du christianisme traditionnellement dominant au Québec et au-delà de la vision étroite qu'ont entretenu à l'endroit de la religion de nombreux thérapeutes-psychanalystes et autres. L'immigration nous confronte non seulement à d'autres cultures et religions mais aussi à d'autres visions de la dimension religieuse. De même, la personne convertie et celle qui demeure enracinée dans sa tradition religieuse nous confrontent comme intervenant et nous forcent peut-être à remettre en question nos conceptions de la croyance religieuse. Pour certains, elle fait partie intégrante de la vie et est un élément incontournable dans l'élaboration d'un plan de traitement. Pour d'autres, elle est un sujet très privé qu'on ne souhaite pas aborder dans une psychothérapie. Enfin, pour les personnes qui intègrent des mouvements sectaires et fondamentalistes, elle peut motiver un renoncement à des valeurs centrales de la modernité telle l'autonomie et ainsi heurter l'intervenant.

Que ce soit dans la clarification de sa propre position face à la religion et à la spiritualité, que ce soit dans la place qu'il lui fait ou non

69. E. Corin, "Le travail du religieux dans la psychose ", Nouveau dialogue, 134 (2001) p. 6-9. 
dans l'évaluation et le traitement ou que ce soit dans le discernement qu'il exerce avant d'orienter son attitude face à tel ou tel patient, l'intervenant a de plus en plus de chances de se retrouver devant des situations complexes qui exigeront de lui davantage de lucidité sur lui-même et de connaissances sur les religions et les spiritualités aussi bien traditionnelles qu'émergentes.

\begin{abstract}
RÉSUMÉ
L'intervenant, qu'il soit psychologue, travailleur social, médecin, ou infirmière, devra éventuellement tenir compte de la dimension religieuse dans le cadre de son travail. Or, il est reconnu que la question religieuse est délicate et qu'elle peut compromettre la démarche de relation d'aide. L'auteur démontre l'importance, pour l'intervenant psycho-social, de se familiariser avec la dimension religieuse. Il examine les différentes positions que celui-ci peut adopter et la nécessité pour lui d'être au clair avec l'ancrage culturel de ses propres pratiques. Il dégage enfin des situations types dans lesquelles peuvent émerger les questions religieuse et spirituelle au Québec.
\end{abstract}

\begin{abstract}
Nowadays, it is probable that health and human services professionals will be confronted with religious and spiritual diversity in their intervention. It is also recognized that the religious issue is a delicate one, to the point that it can jeopardize the therapeutic relationship. The author demonstrates the importance, for the practitioner, to get acquainted with religious and spiritual issues. Different attitudes towards these issues are also examined. They range from religious integration in the therapeutic intervention to the distinctions of these two fields. In all these attitudes, the necessity for personal clarification is emphasized so that the professional's biases and personal beliefs are not in the way of a successful intervention. Finally, typical situations are described where religious and spiritual issues can emerge in the specific context of Québec.
\end{abstract}

